\title{
Comparison of antibody responses to human papillomavirus vaccination as measured by three assays
}

\author{
Hilary A. Robbins ${ }^{1}{ }^{*}$, Troy J. Kemp ${ }^{2}$, Carolina Porras ${ }^{3}$, Ana Cecilia Rodriguez ${ }^{3}$, Mark Schiffman ${ }^{1}$, \\ Sholom Wacholder ${ }^{1}$, Paula Gonzalez ${ }^{3,4}$, John Schiller ${ }^{5}$, Douglas Lowy ${ }^{5}$, Sylviane Poncelet ${ }^{6}$, Mark Esser ${ }^{7}$, \\ Katie Matys ${ }^{8}$, Allan Hildesheim ${ }^{1}$, Ligia A. Pinto ${ }^{2}$, Rolando Herrero ${ }^{3,4}$ and Mahboobeh Safaeian ${ }^{1}$ \\ 1 Division of Cancer Epidemiology and Genetics, National Cancer Institute, NIH, Rockville, MD, USA \\ ${ }^{2}$ HPV Immunology Laboratory, SAIC-Frederick Inc., Frederick National Laboratory for Cancer Research, Frederick, MD, USA \\ ${ }^{3}$ Proyecto Epidemiológico Guanacaste, Fundación INCIENSA, Guanacaste, Costa Rica \\ ${ }^{4}$ International Agency for Research on Cancer, Lyon, France \\ ${ }^{5}$ Center for Cancer Research, National Cancer Institute, NIH, Bethesda, MD, USA \\ ${ }^{6}$ GlaxoSmithKline Biologicals, Rixensart, Belgium \\ 7 Medlmmune, Gaithersburg, MD, USA \\ \& PPD Vaccines and Biologics Center of Excellence, Wayne, PA, USA
}

\section{Edited by:}

Farin Kamangar, Morgan State

University, USA

Reviewed by:

Martha Jane Felini, University of North Texas Health Science Center, USA

Maria Paula Curado, International Prevention Research Institute, France

\section{*Correspondence:}

Hilary A. Robbins, Infections and Immunoepidemiology Branch,

Division of Cancer Epidemiology and Genetics, National Cancer Institute, NIH, 9609 Medical Center Drive, 6-E228, Bethesda, MD 20892, USA e-mail: hilary.robbins@nih.gov
Background: Different assays, including the competitive Luminex immunoassay (cLIA), secreted alkaline phosphatase neutralization assay (SEAP-NA), and virus-like particle-based ELISA, are commonly used to measure antibody responses after human papillomavirus (HPV) vaccination. Direct assay comparisons aid interpretation of immunogenicity data evaluated by different assays.

Methods: We compared CLIA to SEAP-NA and ELISA among 51 HPV16/18-vaccinated women enrolled in the Costa Rica Vaccine Trial. We tested replicate serum samples collected at months 0,1 , and 12 by HPV16/18 cLIA, SEAP-NA, and ELISA. For a subset $(N=10)$, we further tested month 6, 24 and 36 samples. We calculated seroprevalence estimates and Spearman rank correlation coefficients comparing CLIA to SEAP-NA and ELISA.

Results: After one vaccine dose, seroprevalence by SEAP-NA and ELISA was 100\% (both HPV16 and HPV18), and by CLIA was 96\% (95\% Cl 87-100\%) for HPV16 and $71 \%(95 \%$ Cl 56-83\%) for HPV18. Seroprevalence was $100 \%$ by all assays after three doses. Correlation between assays was high after one vaccine dose [CLIA/SEAP-NA $\rho=0.91$ (HPV16) and $\rho=0.86$ (HPV18); cLIA/ELISA $\rho=0.84$ (HPV16) and $\rho=0.74$ (HPV18); all $p<0.001$ ] and remained high through month 36 . Ratios of mean antibody levels to seropositivity cutoffs at month 36 were lower for CLIA than for SEAP-NA or ELISA, particularly for HPV18 (HPV18 ratio for CLIA 1.9, SEAP-NA 3.5, ELISA 3.4).

Conclusion: Though correlation between cLIA and SEAP-NA/ELISA is high and stable after vaccination, the assays differ in scale and sensitivity, with notable differences after one vaccine dose and for HPV18. Our results demonstrate that comparisons of antibody responses to HPV vaccination measured by different assays are approximate, and must consider biological and technical differences between assays.

Keywords: human papillomavirus, HPV serology, HPV vaccines, cLIA, SEAP-NA, ELISA

\section{INTRODUCTION}

Two human papillomavirus (HPV) virus-like particle (VLP) vaccines, the bivalent (HPV16/18) Cervarix ${ }^{\circledR}$ and quadrivalent (HPV6/11/16/18) Gardasil ${ }^{\circledR}$, are licensed for prevention of cervical cancer and related lesions (1). Four-year efficacy of both vaccines approaches $100 \%$ among HPV-naïve women for prevention of high-grade lesions related to HPV types 16 and $18(2,3)$, which together cause $70 \%$ of cervical cancers (4).

Neutralizing antibody responses are believed to be the primary mechanism of vaccine-induced protection (5), but different type-specific assays are used to measure them. For Cervarix ${ }^{\circledR}$, the principal assay for measuring immunogenicity has been the VLPbased ELISA, which measures neutralizing and non-neutralizing antibodies of one immunoglobulin class (typically $\mathrm{IgG}$ ). Neutralizing responses to Cervarix ${ }^{\circledR}$ have been measured using the secreted alkaline phosphatase neutralization assay (SEAP-NA), which broadly and directly measures neutralization potential (6). For Gardasil ${ }^{\circledR}$, the proprietary competitive Luminex immunoassay (cLIA) is primarily used, which measures neutralizing antibodies that compete for binding to one VLP epitope (7).

Measured antibody responses are important, as they are used as evidence of vaccine immunogenicity, but results vary by assay. 
When different vaccines are evaluated using different assays, variability in results may be due to assay differences or due to true differences in immunogenicity (8). To facilitate interpretation of measurements by different assays, direct comparisons of assays have been published in both natural infection and vaccination contexts (9-12). A previous report by our group compared SEAP-NA to ELISA after vaccination with Cervarix ${ }^{\circledR}$, finding high correlation between the two assays (10). Here, we extend these results by presenting a detailed and longitudinal post-vaccination comparison of cLIA to SEAP-NA and ELISA.

\section{MATERIALS AND METHODS}

We evaluated women selected from the HPV vaccine arm of the Costa Rica Vaccine Trial (CVT) (13), in which participants were vaccinated with Cervarix ${ }^{\circledR}$ at months 0,1 , and 6 . For the present study, we combined two groups of women sampled for previous serological studies where HPV16/18 SEAP-NA and ELISA testing were already performed $(10,14,15)$. The first group included 50 randomly sampled women, and the second group included 12 women sampled with the requirement of no cervical infection with HPV types $16,18,31,45$, or 58 at baseline (month 0 ). We further tested replicate sera from these 62 women collected at months 0 , 1 , and 12 by HPV16/18 cLIA; for the smaller group, we additionally tested samples from months 6, 24 and 36. We excluded four women who received fewer than three vaccine doses and five for whom any of the assays failed, and further excluded two women with anomalous results in post-vaccination samples. One woman was seronegative by all assays at month 12 despite a normal antibody response at other time points; we suspect this may reflect a sample retrieval error. The second woman never seroconverted by HPV16/18 cLIA after vaccination; this may indicate a technical error but does not imply a vaccine failure as other assays showed the expected antibody response. As results did not differ by group, we conducted analyses among a combined analytical sample of 51 women, equal to 62 less 11 exclusions.

The SEAP-NA was performed at the HPV Immunology Laboratory, SAIC-Frederick Inc., as described $(6,10)$, with results reflecting the mean of at least duplicate testing (maximum of 15 runs) for each sample. Neutralization titers were calculated by linear interpolation and were defined as the reciprocal of the dilution that reduced SEAP activity by $50 \%$ compared to control wells. We used the laboratory-determined seropositivity cutoff of 10, which reflects our lowest dilution. The VLP-based ELISA was performed at GSK Biologicals as described (16), with results reflecting the mean of between 1 and 5 runs per sample, and laboratory-determined seropositivity cutoffs of $8 \mathrm{EU} / \mathrm{mL}$ (ELISA units per milliliter) (HPV16) and $7 \mathrm{EU} / \mathrm{mL}$ (HPV18) were used. Data describing our ELISA (10) and SEAP-NA $(10,14,15)$ results have been published. The cLIA was performed at PPD Vaccines and Biologicals as described (7), with use of standard curves to convert mean fluorescence intensities to arbitrary milli-Merck units per milliliter (mMU/mL). Laboratory-determined cutoffs of $20 \mathrm{mMU} / \mathrm{mL}$ (HPV16) and $24 \mathrm{mMU} / \mathrm{mL}$ (HPV18) are based on an algorithm maximizing the distinction between "likely negative" and "likely positive" samples (17).

We performed statistical analyses separately for HPV16 and HPV18. Among the combined sample of 51 women, at months
0,1 , and 12 , we compared cLIA to SEAP-NA and ELISA using seroprevalence estimates, scatter plots, and Spearman rank correlation coefficients. Among the subgroup of 10 women (12 less 2 exclusions), where we additionally had assay data at months 6,24 and 36, we plotted individual antibody levels by each assay over time. To quantify inter-assay correlation beyond month 12 (i.e., beyond time points available in the larger sample), we also calculated Spearman coefficients at month 36 among these 10 women. For graphical presentation and calculation of correlation coefficients, values below assay lower limits of detection (LLODs) were assigned a value of 1/2 (LLOD).

\section{RESULTS}

HPV16/18 antibody levels increased after vaccination as previously reported by SEAP-NA and ELISA $(10,14,15)$ and by cLIA (Figures 1 and 2). At 1 month after the first vaccine dose, all women were HPV16 and HPV18 seropositive by SEAP-NA and ELISA (Table 1). By cLIA, at month 1, 96\% (95\% CI 87-100\%) and 71\% (95\% CI 56-83\%) were seropositive for HPV16 and HPV18, respectively. At month 12, all women were seropositive for both HPV types by all three assays.

Prior to vaccination, when average antibody levels were very low or below detection limits, correlation between cLIA and SEAP-NA was moderate for HPV16 $(\rho=0.39, p=0.004$, Figure 1A) and not present for HPV18 $(\rho=-0.04, p=0.774$, Figure 1C). Results were similar for CLIA and ELISA $(\rho=0.58, p<0.001$ for HPV 16; $\rho=-0.07, p=0.629$ for HPV18, Figures 1B,D). Correlation was high after one vaccine dose (i.e., at month 1), with Spearman coefficients of 0.91 (HPV16) and 0.86 (HPV18) for cLIA and SEAP-NA, and coefficients of 0.84 (HPV16) and 0.74 (HPV18) for CLIA and ELISA (all $p<0.001$ ). Correlation remained high 6 months after the third dose (i.e., at month 12), with Spearman coefficients of 0.79 (HPV16) and 0.87 (HPV18) for cLIA and SEAP-NA, and coefficients of 0.81 (HPV16) and 0.86 (HPV18) for CLIA and ELISA (all $p<0.001$ ). Discordant samples (i.e., samples seronegative by one assay but seropositive by the other) were present only at months 0 and 1, and were seronegative by cLIA but seropositive by either SEAP-NA or ELISA in all cases (Figure 1). Using data at month $36(N=10)$, when plateau antibody levels are expected, correlation coefficients were 0.89 (HPV16, $p=0.001$ ) and 0.83 (HPV18, $p=0.003$ ) for CLIA and SEAP-NA, and 0.96 $($ HPV16, $p<0.001)$ and 0.92 (HPV18, $p<0.001)$ for cLIA and ELISA (results not shown).

Antibody kinetics throughout and after vaccination were largely similar across HPV types, assays, and the 10 women studied (Figure 2), with increases in antibody levels throughout vaccination and a gradual decline after month 12. For HPV16/18 SEAP-NA and ELISA, levels were well above seropositivity cutoffs beginning at month 1 (Figures 2C-F). For cLIA, particularly for HPV18, levels were lower and closer to the seropositivity cutoff (Figures 2A,B). To quantify this observation at our final time point (month 36), we calculated the ratio of the mean of log-antibody levels to the corresponding log-cutoff (log-antibodymean: $\log$-cutoff). For HPV16, these ratios were 2.4 for CLIA, 3.4 for SEAP-NA, and 3.6 for ELISA. For HPV18, these ratios were 1.9 for CLIA, 3.5 for SEAP-NA, and 3.4 for ELISA. 

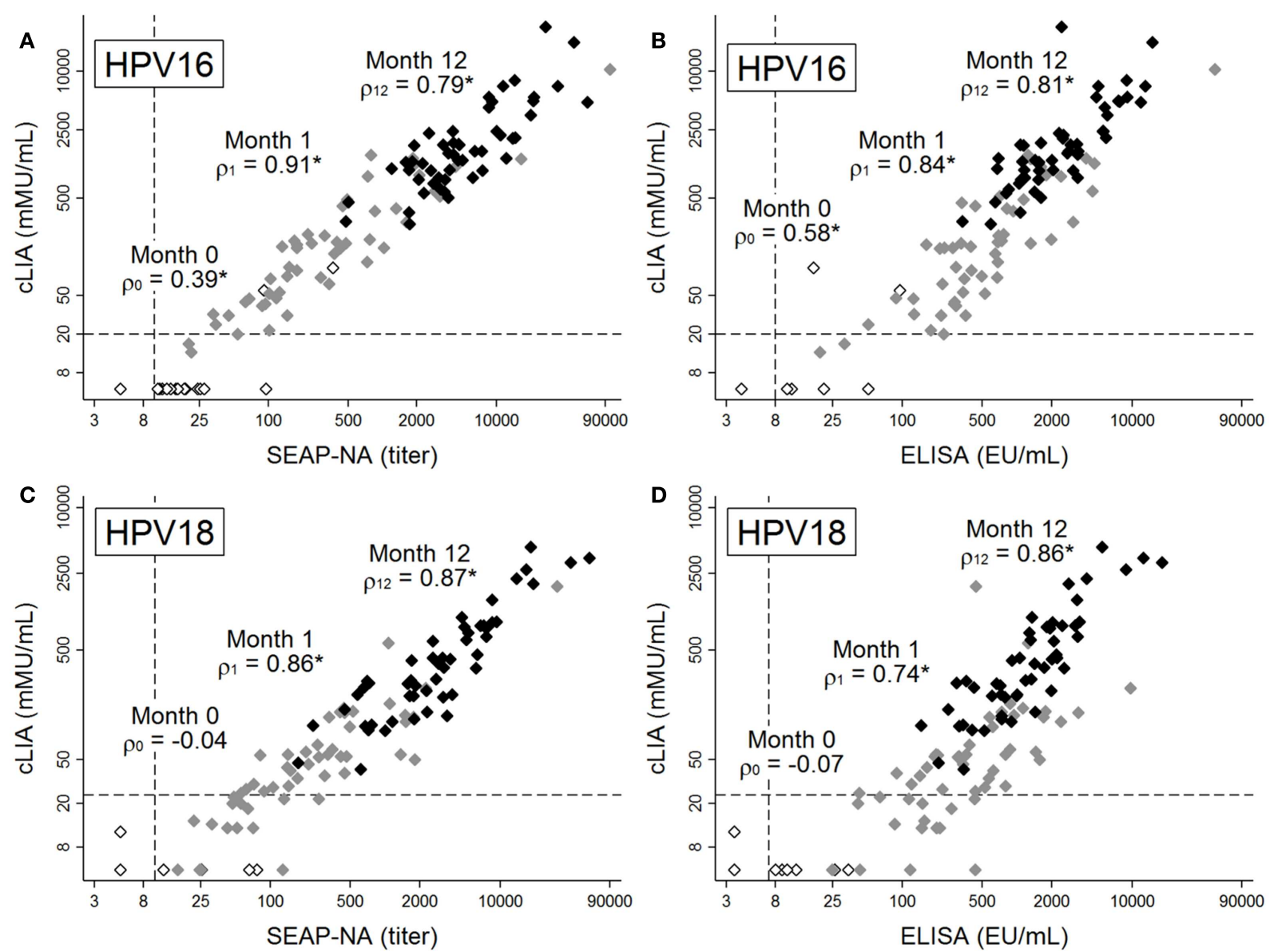

FIGURE 1 | Antibody levels for HPV16 (A,B) and HPV18 (C,D), as measured by cLIA vs. SEAP-NA $(A, C)$ or cLIA vs. ELISA (B,D). Levels were measured at months 0 (white markers), 1 (gray markers), and 12 (black markers) among 51 women in the Costa Rica Vaccine Trial who received HPV vaccine doses at months 0,1 , and 6 . Spearman rank

\section{DISCUSSION}

Different assays are used to monitor antibody responses following HPV vaccination, with ELISA or SEAP-NA typically used for Cervarix $^{\circledR}$ and cLIA for Gardasil ${ }^{\circledR}$. To aid interpretation of immunogenicity data, we directly compared cLIA-measured responses to ELISA- and SEAP-NA-measured responses after vaccination with Cervarix ${ }^{\circledR}$. Correlation between cLIA and both SEAP-NA and ELISA was high beginning after one vaccine dose, with similar antibody kinetics across assays. However, levels relative to seropositivity cutoffs were lower by cLIA than by SEAP-NA or ELISA, particularly for HPV18.

The cLIA, SEAP-NA, and ELISA are technically different assays and measure different aspects of the antibody response (8). The cLIA measures neutralizing antibodies of all immunoglobulin classes that compete for binding to a specific VLP epitope (V5 for HPV16, J4 for HPV18) by evaluating the strength of a fluorescent signal produced by binding monoclonal antibodies (7). SEAPNA measures a reporter gene product which is expressed when HPV pseudovirions infect susceptible cells, such that decreases in correlation coefficients between pairs of assays are displayed for each time point, with asterisks denoting statistical significance (all $p<0.005$ ) Dashed lines represent laboratory-determined seropositivity cutoffs. Note differing scales for $x$-and $y$-axes, as assays use different scales for measurement.

expression reflect overall serum neutralizing potential (6), which is assumed but not required to be antibody-mediated. ELISA measures one class of antibodies (typically IgG) that bind to a fixed VLP antigen by measuring the activity of an enzyme conjugated to a secondary antibody (16). These assays use different scales in terms of measurement, and further differences exist in the structure and production of the VLPs for each assay. The directed nature and more stringent cutoff of cLIA affords it lower sensitivity than SEAP-NA or ELISA (9); further, within the cLIA, the HPV18 J4 epitope may be less immunodominant than the HPV16 V5 epitope.

In our study, the lower sensitivity of cLIA was likely responsible for a number of discordant samples after vaccination. At month 1, approximately $4 \%$ of samples were HPV 16 seropositive by ELISA and SEAP-NA but HPV16 seronegative by cLIA. The proportion of discordant samples was larger (29\%) for HPV18 at the same visit, likely reflecting lower immunodominance of the HPV18 J4 epitope than the HPV16 V5 epitope. Relative to seropositivity cutoffs, HPV18 antibody levels were substantially lower by cLIA than by SEAP-NA or ELISA. 


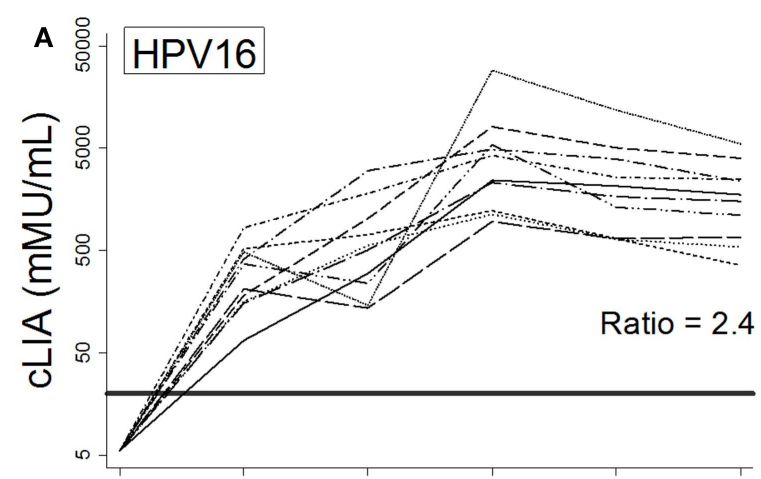

Month 0 Month 1 Month 6 Month 12 Month 24 Month 36 Visit

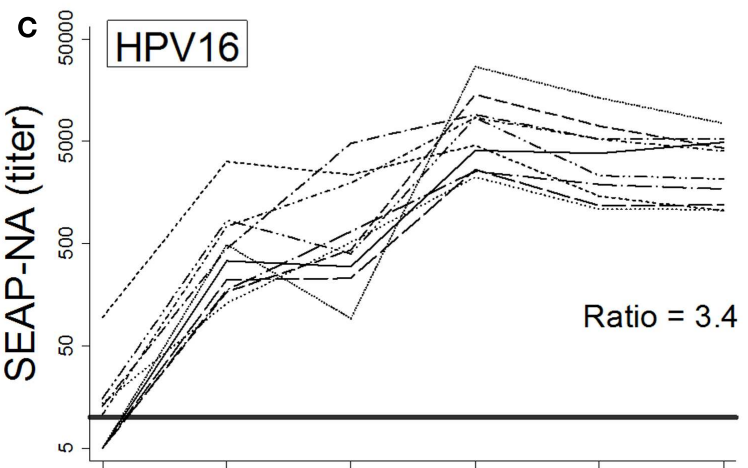

Month 0 Month 1 Month 6 Month 12 Month 24 Month 36 Visit

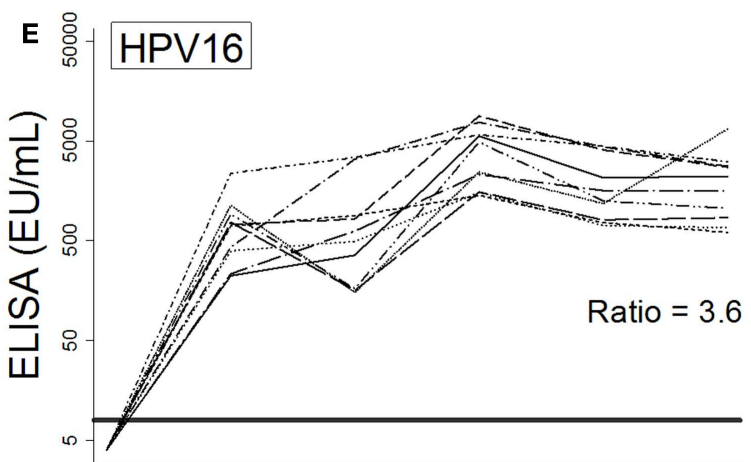

Month 0 Month 1 Month 6 Month 12 Month 24 Month 36 Visit

FIGURE 2 | Individual patterns of antibody levels over time as measured by $\operatorname{cLIA}(A, B)$, SEAP-NA $(C, D)$, and ELISA $(E, F)$ among 10 women in the Costa Rica Vaccine Trial who received HPV vaccine doses at months 0,1 , and 6. Patterns are shown for HPV16 (A,C,E) and HPV18 (B,D,F). Bold solid

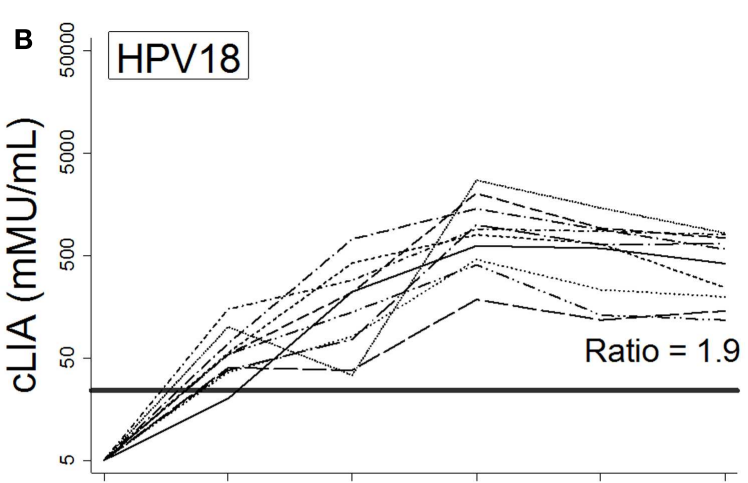

Month 0 Month 1 Month 6 Month 12 Month 24 Month 36 Visit

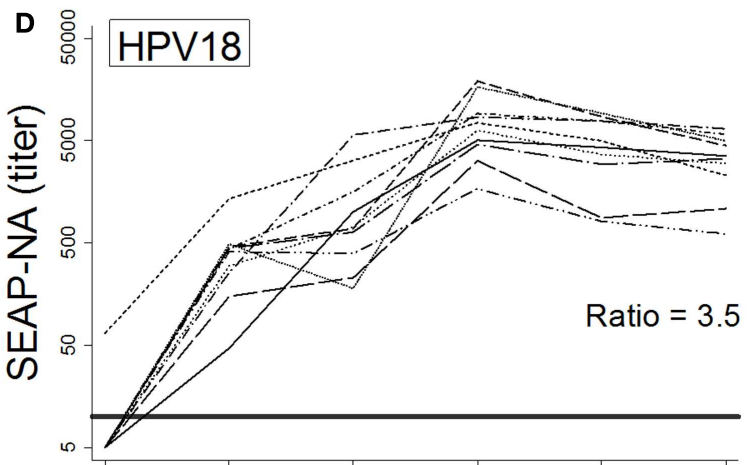

Month 0 Month 1 Month 6 Month 12 Month 24 Month 36 Visit

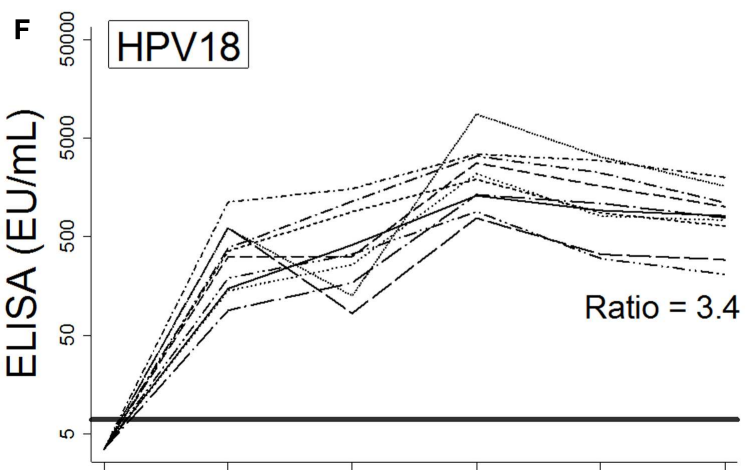

Month 0 Month 1 Month 6 Month 12 Month 24 Month 36 Visit

lines represent seropositivity cutoffs, and ratios at 36 months were calculated as the mean of log-antibody levels to the log of the corresponding seropositivity cutoff. HPV DNA negativity for types 16, 18, 31, 45, and 58 at month 0 was required for selection.
Despite assay differences, we observed high and statistically significant correlation between cLIA and both SEAP-NA and ELISA beginning after one vaccine dose and extending to month 36, with similar antibody kinetics across HPV types and assays. A previous study, comparing cLIA to an in-house SEAP-NA, found correlation coefficients of 0.67 (HPV16) and 0.91 (HPV18) 1 month after the third dose of Gardasil ${ }^{\circledR}$ (12). Another study conducted among Gardasil ${ }^{\circledR}$ vaccinees compared cLIA to a total
IgG Luminex immunoassay, which is biologically similar to but technically different from our ELISA (18). Results were similar to our cLIA/ELISA comparison, with correlation coefficients at months 7, 24, and 48 of $0.66,0.90$, and 0.91 for HPV16, and 0.84, 0.88 , and 0.89 for HPV18. Our group previously reported high correlation between SEAP-NA and ELISA for combined 1- and 12 -month measurements, with coefficients of 0.91 (HPV16) and 0.85 (HPV18) (10). 
Table 1 | Seroprevalence by HPV type, assay, and visit among 51 women in the Costa Rica Vaccine Trial.

\begin{tabular}{lllll}
\hline HPV type & Assay & \multicolumn{2}{l}{$\begin{array}{l}\text { Seroprevalence and 95\% confidence } \\
\text { interval }\end{array}$} \\
\cline { 3 - 5 } & & Month $\mathbf{~}$ & Month $\mathbf{1}$ & Month 12 \\
\hline HPV16 & CLIA & $4(0-13)$ & $96(87-100)$ & $100(93-100)$ \\
& SEAP-NA & $33(21-48)$ & $100(93-100)$ & $100(93-100)$ \\
& ELISA & $12(4-24)$ & $100(93-100)$ & $100(93-100)$ \\
HPV18 & CLIA & $0(0-7)$ & $71(56-83)$ & $100(93-100)$ \\
& SEAP-NA & $8(2-19)$ & $100(93-100)$ & $100(93-100)$ \\
& ELISA & $20(10-33)$ & $100(93-100)$ & $100(93-100)$ \\
& & & &
\end{tabular}

Seropositivity was defined according to laboratory cutoffs (see Materials and Methods). HPV vaccine doses were administered at months 0,1 , and 6 .

${ }^{a}$ For estimates where seroprevalence is 0 or $100 \%$, a one-sided $97.5 \%$ confidence interval is shown.

In conclusion, though agreement between assays is not high prior to vaccination (9), among vaccinated women measurements by cLIA, SEAP-NA, and ELISA correlate well and have similar patterns over time. However, the assays differ in scale and sensitivity, with notable differences after one vaccine dose and for HPV18. Comparisons of antibody responses to HPV vaccination measured by different assays are therefore approximate, and must consider biological and technical differences between assays.

\section{ACKNOWLEDGMENTS}

\section{Funding}

The Costa Rica HPV Vaccine Trial is a long-standing collaboration between investigators in Costa Rica and the National Cancer Institute (NCI). The trial is sponsored and funded by the NCI (contract N01-CP-11005), with funding support from the National Institutes of Health Office of Research on Women's Health, and done with the support from the Ministry of Health of Costa Rica. Vaccine was provided for our trial by GlaxoSmithKline Biologicals, under a Clinical Trials Agreement with the NCI. GlaxoSmithKline also provided support for aspects of the trial associated with regulatory submission needs of the company under US Food and Drug Administration BB-IND 7920.

\section{Investigators in the Costa Rica Vaccine Trial (CVT) group} Proyecto Epidemiológico Guanacaste, Fundación INCIENSA, San José, Costa Rica: Mario Alfaro (cytopathologist), Manuel Barrantes (field supervisor), M. Concepción Bratti (co-investigator), Fernando Cárdenas (general field supervisor), Bernal Cortés (specimen and repository manager), Albert Espinoza (head, coding, and data entry), Yenory Estrada (pharmacist), Paula González (co-investigator), Diego Guillén (pathologist), Roland Herrero (co-principal investigator), Silvia E. Jiménez (trial coordinator), Jorge Morales (colposcopist), Luis Villegas (colposcopist), Lidia Ana Morera (head study nurse), Elmer Pérez (field supervisor), Carolina Porras (co-investigator), Ana Cecilia Rodríguez (co-investigator), Libia Rivas (clinical coordinator). University of Costa Rica, San José, Costa Rica: Enrique Freer (director, HPV Diagnostics Laboratory), José Bonilla (head, HPV Immunology
Laboratory), Alfanso García-Piñeres (immunologist), Sandra Silva (head microbiologist, HPV Diagnostics Laboratory), Ivannia Atmella (microbiologist, Immunology Laboratory), Margarita Ramírez (microbiologist, Immunology Laboratory). United States National Cancer Institute, Bethesda, MD, USA: Allan Hildesheim (co-principal investigator and NCI co-project officer), Hormuzd Katki (statistician), Aimée R. Kreimer (co-investigator), Douglas R. Lowy (HPV virologist), Nora Macklin (trial coordinator), Mark Schiffman (medical monitor and NCI co-project officer), John T. Schiller (HPV virologist), Mark Sherman (QC pathologist), Diane Solomon (medical monitor and QC pathologist), Sholom Wacholder (statistician). SAIC, NCI-Frederick, Frederick, $M D$, USA: Ligia Pinto (head, HPV immunology laboratory), Troy Kemp (immunologist). Women's and Infants' Hospital, Providence, RI, USA: Claire Eklund (QC cytology), Martha Hutchinson (QC cytology). Georgetown University, Washington, DC, USA: Mary Sidawy (histopathologist). DDL Diagnostic Laboratory, Netherlands: Wim Quint (virologist, HPV DNA testing), Leen-Jan van Doorn (HPV DNA testing).

\section{Information Management Services}

Brian Befano (data management programmer).

\section{REFERENCES}

1. Schiller JT, Castellsague X, Garland SM. A review of clinical trials of human papillomavirus prophylactic vaccines. Vaccine (2012) 30(Suppl 5):F123-38. doi:10.1016/j.vaccine.2012.04.108

2. Munoz N, Kjaer SK, Sigurdsson K, Iversen OE, Hernandez-Avila M, Wheeler $\mathrm{CM}$, et al. Impact of human papillomavirus (HPV)-6/11/16/18 vaccine on all HPV-associated genital diseases in young women. J Natl Cancer Inst (2010) 102:325-39. doi:10.1093/jnci/djp534

3. Lehtinen M, Paavonen J, Wheeler CM, Jaisamrarn U, Garland SM, Castellsague X, et al. Overall efficacy of HPV-16/18 AS04-adjuvanted vaccine against grade 3 or greater cervical intraepithelial neoplasia: 4-year end-of-study analysis of the randomised, double-blind PATRICIA trial. Lancet Oncol (2012) 13:89-99. doi:10.1016/S1470-2045(11)70286-8

4. Lowy DR, Schiller JT. Reducing HPV-associated cancer globally. Cancer Prev Res (Phila) (2012) 5:18-23. doi:10.1158/1940-6207.CAPR-11-0542

5. Schiller JT, Lowy DR. Understanding and learning from the success of prophylactic human papillomavirus vaccines. Nat Rev Microbiol (2012) 10:681-92. doi:10.1038/nrmicro2872

6. Pastrana DV, Buck CB, Pang YY, Thompson CD, Castle PE, FitzGerald PC, et al. Reactivity of human sera in a sensitive, high-throughput pseudovirus-based papillomavirus neutralization assay for HPV16 and HPV18. Virology (2004) 321:205-16. doi:10.1016/j.virol.2003.12.027

7. Opalka D, Lachman CE, MacMullen SA, Jansen KU, Smith JF, Chirmule N, et al. Simultaneous quantitation of antibodies to neutralizing epitopes on virus-like particles for human papillomavirus types 6,11,16, and 18 by a multiplexed luminex assay. Clin Diagn Lab Immunol (2003) 10:108-15. doi:10.1128/CDLI.10.1.108-115.2003

8. Schiller JT, Lowy DR. Immunogenicity testing in human papillomavirus viruslike-particle vaccine trials. J Infect Dis (2009) 200:166-71. doi:10.1086/599988

9. Safaeian M, Ghosh A, Porras C, Lin SW, Rodriguez AC, Schiffman M, et al. Direct comparison of HPV16 serological assays used to define HPV-naive women in HPV vaccine trials. Cancer Epidemiol Biomarkers Prev (2012) 21:1547-54. doi:10.1158/1055-9965.EPI-12-0558

10. Kemp TJ, Garcia-Pineres A, Falk RT, Poncelet S, Dessy F, Giannini SL, et al. Evaluation of systemic and mucosal anti-HPV16 and anti-HPV18 antibody responses from vaccinated women. Vaccine (2008) 26:3608-16. doi:10.1016/j. vaccine.2008.04.074

11. Lin SW, Ghosh A, Porras C, Markt SC, Rodriguez AC, Schiffman M, et al. HPV16 seropositivity and subsequent HPV16 infection risk in a naturally infected population: comparison of serological assays. PLoS One (2013) 8:e53067. doi:10.1371/journal.pone.0053067 
12. Krajden M, Cook D, Yu A, Chow R, Mei W, McNeil S, et al. Human papillomavirus 16 (HPV 16) and HPV 18 antibody responses measured by pseudovirus neutralization and competitive Luminex assays in a two- versus three-dose HPV vaccine trial. Clin Vaccine Immunol (2011) 18:418-23. doi:10.1128/CVI. 00489- 10

13. Herrero R, Hildesheim A, Rodriguez AC, Wacholder S, Bratti C, Solomon D, et al Rationale and design of a community-based double-blind randomized clinical trial of an HPV 16 and 18 vaccine in Guanacaste, Costa Rica. Vaccine (2008) 26:4795-808. doi:10.1016/j.vaccine.2008.07.002

14. Kemp TJ, Hildesheim A, Safaeian M, Dauner JG, Pan Y, Porras C, et al. HPV16/18 L1 VLP vaccine induces cross-neutralizing antibodies that may mediate crossprotection. Vaccine (2011) 29:2011-4. doi:10.1016/j.vaccine.2011.01.001

15. Kemp TJ, Safaeian M, Hildesheim A, Pan Y, Penrose KJ, Porras C, et al. Kinetic and HPV infection effects on cross-type neutralizing antibody and avidity responses induced by Cervarix((R)). Vaccine (2012) 31:165-70. doi:10.1016/ j.vaccine.2012.10.067

16. Dessy FJ, Giannini SL, Bougelet CA, Kemp TJ, David MP, Poncelet SM, et al. Correlation between direct ELISA, single epitope-based inhibition ELISA and pseudovirion-based neutralization assay for measuring anti-HPV-16 and antiHPV-18 antibody response after vaccination with the AS04-adjuvanted HPV16/18 cervical cancer vaccine. Hum Vaccin (2008) 4:425-34. doi:10.4161/hv.4.6. 6912

17. Dias D, Van DJ, Schlottmann S, Kelly S, Puchalski D, Ruiz W, et al. Optimization and validation of a multiplexed luminex assay to quantify antibodies to neutralizing epitopes on human papillomaviruses 6,11, 16, and 18. Clin Diagn Lab Immunol (2005) 12:959-69. doi:10.1128/CDLI.12.8.959-969.2005

18. Brown DR, Garland SM, Ferris DG, Joura E, Steben M, James M, et al. The humoral response to Gardasil over four years as defined by total IgG and competitive Luminex immunoassay. Hum Vaccin (2011) 7:230-8. doi:10.4161/ hv.7.2.13948xs

Conflict of Interest Statement: One or more of the authors is employed by a commercial company. These include: Sylviane Poncelet, GlaxoSmithKline Biologicals; Mark Esser, MedImmune; Katie Matys, PPD Vaccines and Biologics. John Schiller and Douglas Lowy report that they are named inventors on US Government-owned HPV vaccine patents that are licensed to GlaxoSmithKline and Merck and for which the National Cancer Institute receives licensing fees. They are entitled to limited royalties as specified by federal law. The other authors declare that they have no conflicts of interest.

Received: 26 November 2013; accepted: 20 December 2013; published online: 13 January 2014.

Citation: Robbins HA, Kemp TJ, Porras C, Rodriguez AC, Schiffman M, Wacholder S, Gonzalez P, Schiller J, Lowy D, Poncelet S, Esser M, Matys K, Hildesheim A, Pinto LA, Herrero $R$ and Safaeian $M$ (2014) Comparison of antibody responses to human papillomavirus vaccination as measured by three assays. Front. Oncol. 3:328. doi: $10.3389 /$ fonc. 2013.00328

This article was submitted to Cancer Epidemiology and Prevention, a section of the journal Frontiers in Oncology.

Copyright (c) 2014 Robbins, Kemp, Porras, Rodriguez, Schiffman, Wacholder, Gonzalez, Schiller, Lowy, Poncelet, Esser, Matys, Hildesheim, Pinto, Herrero and Safaeian. This is an open-access article distributed under the terms of the Creative Commons Attribution License (CC BY). The use, distribution or reproduction in other forums is permitted, provided the original author(s) or licensor are credited and that the original publication in this journal is cited, in accordance with accepted academic practice. No use, distribution or reproduction is permitted which does not comply with these terms. 